\title{
Challenges in the Laboratory Diagnosis and Clinical Management of Heteroresistant Vancomycin Staphylococcus aureus (hVISA)
}

Yamuna Devi ${ }^{1}$, Punithavathy PM $^{1}$, Sangeeta Thomas ${ }^{2}$ and Balaji Veeraraghavan ${ }^{1 *}$

${ }^{1}$ Department of Clinical Microbiology, Christian Medical College, Vellore, Tamil Nadu, India

${ }^{2}$ Travancore Medical College, Kollam, India

"Corresponding author: Balaji Veeraraghavan, Department of Clinical Microbiology, Christian Medical College, Vellore, Tamil Nadu, 632 004, India, Tel: +91-9442210555; E-mail: vbalaji@cmcvellore.ac.in

Received date: June 24, 2015; Accepted date: August 12, 2015; Published date: August 19, 2015

Copyright: ( 2015 Devi Y, et al. This is an open-access article distributed under the terms of the Creative Commons Attribution License, which permits unrestricted use, distribution, and reproduction in any medium, provided the original author and source are credited.

\begin{abstract}
MRSA is a public health threat and significant cause of health care-associated and community-associated infections. Vancomycin is considered as gold standard in treating life-threatening infections. Recently, reduced vancomycin susceptibility has been reported across the world. However, infection with $S$. aureus resistant to vancomycin is clinically rare and less relevant in causing infections. The clinical failure of vancomycin therapy is being increasingly reported with vancomycin intermediate S. aureus (VISA) and hetero-resistant vancomycin intermediate $S$. aureus (hVISA). The therapeutic option in treating hVISA and VISA remains uncertain. Extensive use of vancomycin over a period of time leads to generation of selective pressure and emergence of resistance. In addition, vancomycin has low tissue penetration, slow bactericidal activity and risk of nephrotoxicity. Although, phenotypic feature of VISA and hVISA were well known, their genetic basis remains unknown. VISA and hVISA is generated by the accumulation of mutation leads to thickening of cell wall. Trapping of vancomycin molecule occurs due to the presence of false targets D-ala D-ala residues in the cell wall, before reaching its target site in cytoplasmic membrane. Despite, it is difficult to detect hVISA using standard susceptibility testing methods. Treatment failure is common among patients with high bacterial load infections. Treating of patients with hVISA/ VISA infections is of great challenge for clinicians.
\end{abstract}

\section{Introduction}

Vancomycin has remained the mainstay for the treatment of MRSA infections in the past four decades and the emergence of resistance to this drug has been very slow. Vancomycin, a glycopeptide antibiotic, was introduced into clinical practice in 1958 to treat life threatening infections caused by Gram positive organisms. It acts by inhibiting cell wall synthesis by binding to cell wall precursors such as D-ala-D-ala residues. The molecule is large and rigid and hence its binding to the residues causes stearic hindrance which results in inefficient cell wall synthesis [1]. Overuse of vancomycin has led to the development of a selective pressure over time leading to emergence of reduced vancomycin susceptibility among $S$. aureus strains. Since the repertoire of drugs available for treating MRSA, not responding to vancomycin is limited, emergence of MRSA with reduced susceptibility to vancomycin is dangerous and worrisome. This review will summarize the current knowledge on mechanism of resistance, clinical impact and laboratory diagnosis of hVISA

\section{Heteroresistant Vancomycin Intermediate $S$. aureus (hVISA)}

There is no precise definition for heteroresistant vancomycin intermediate $S$. aureus (hVISA). As per CLSI guidelines, hVISA has been found to reside within the susceptible range of MIC $(\leq 2 \mu \mathrm{g} / \mathrm{ml})$ where a notable subpopulation of resistant cells presents approximately at a frequency of 1 in $10^{5}$ cells to $10^{6}$ cells [2]. If the proportion of MRSA in a population expressing vancomycin resistance is sufficiently high, it can be detected with the standard low inocula of susceptibility testing as non-susceptible. However, hVISA subpopulations occur at frequency far below the threshold of standard inoculum and misidentified as susceptible with standard susceptibility testing. Moreover their varying levels of resistance and extremely unstable phenotypic expressions render their detection more complicated [3].

hVISA and VISA share the same mechanism of resistance whereby increased cell wall thickness and high number of D-ala-D-ala residues cause a trapping of the large vancomycin molecules [4] (Table 1). The hVISA is considered as the precursor stage of VISA wherein selection pressure created by sub-therapeutic levels of vancomycin therapy can aid the selective growth of these resistant subpopulations. Prolonged therapy with vancomycin eventually leads to a uniform population of strains expressing VISA.

\section{Molecular Determinants of hVISA and VISA}

Vancomycin binds with pseudotarget or false target (D-ala-D-ala residues) with high affinity cell wall peptidoglycan layer. However, the binding doesn't affect the cell viability. Because the vital target is missed by vancomycin $[5,6]$. Thus, vancomycin hardly penetrates the cell wall into cytoplasmic membrane. Studies have reported the gene expression ad mutation in regulatory genes is crucial for the cause of cell wall thickening and alteration of metabolism. The present knowledge on specific genetic determinants of hVISA and VISA was inadequate.

Currently, understood emergence of hVISA and VISA was reported to occur due to: 
Citation: Devi Y, Punithavathy PM, Thomas S, Veeraraghavan B (2015) Challenges in the Laboratory Diagnosis and Clinical Management of Heteroresistant Vancomycin Staphylococcus aureus (hVISA). Clin Microbiol 4: 214. doi:10.4172/2327-5073.1000214

Page 2 of 9

- mutation in the regulatory genes, this includes; vraSR two component regulatory systems (TCRS) and walKR two component regulatory systems (Figure 1A) and

- $\quad$ rpoB gene mutation (Figure 1B).

\begin{tabular}{|c|c|c|c|}
\hline Characteristics & hVISA & VISA & VRSA \\
\hline MIC & $1-2 \mu \mathrm{g} / \mathrm{ml}$ & $4-8 \mu \mathrm{g} / \mathrm{ml}$ & $\geq 16 \mu \mathrm{g} / \mathrm{ml}$ \\
\hline Mechanism of resistance & $\begin{array}{l}\text { Cell wall thickening and hyperproduction } \\
\text { of glycopeptide binding targets }\end{array}$ & $\begin{array}{c}\text { Cell wall thickening and hyperproduction of } \\
\text { glycopeptide binding targets. }\end{array}$ & $\begin{array}{l}\text { Substitution of D-Ala-D-Ala with D- } \\
\text { Ala-D-Lac }\end{array}$ \\
\hline Gene encoding for resistance & $\begin{array}{l}\text { Endogenous resistance-Chromosomal } \\
\text { mutation }\end{array}$ & $\begin{array}{c}\text { Endogenous resistance-Chromosomal } \\
\text { mutation }\end{array}$ & Van A \\
\hline $\begin{array}{l}\text { Recommended methods for } \\
\text { detection in CLSI guidelines }\end{array}$ & - & $\begin{array}{c}\text { Vancomycin MIC: E-test, Microbroth dilution } \\
\text { method }\end{array}$ & $\begin{array}{l}\text { Vancomycin MIC: E-test, Microbroth } \\
\text { dilution method }\end{array}$ \\
\hline $\begin{array}{l}\text { Recommended methods for } \\
\text { detection in EUCAST guidelines }\end{array}$ & \multicolumn{3}{|c|}{$\begin{array}{l}\text { Screening methods (hVISA, VISA and VRSA): Macro E-test, Glycopeptide resistance detection test and Teicoplanin screening } \\
\text { agar. } \\
\text { Confirmatory testing for hVISA/VISA: Population analysis profile-Gold standard }\end{array}$} \\
\hline
\end{tabular}

Table 1: Characteristics of hVISA, VISA and VRSA.

A

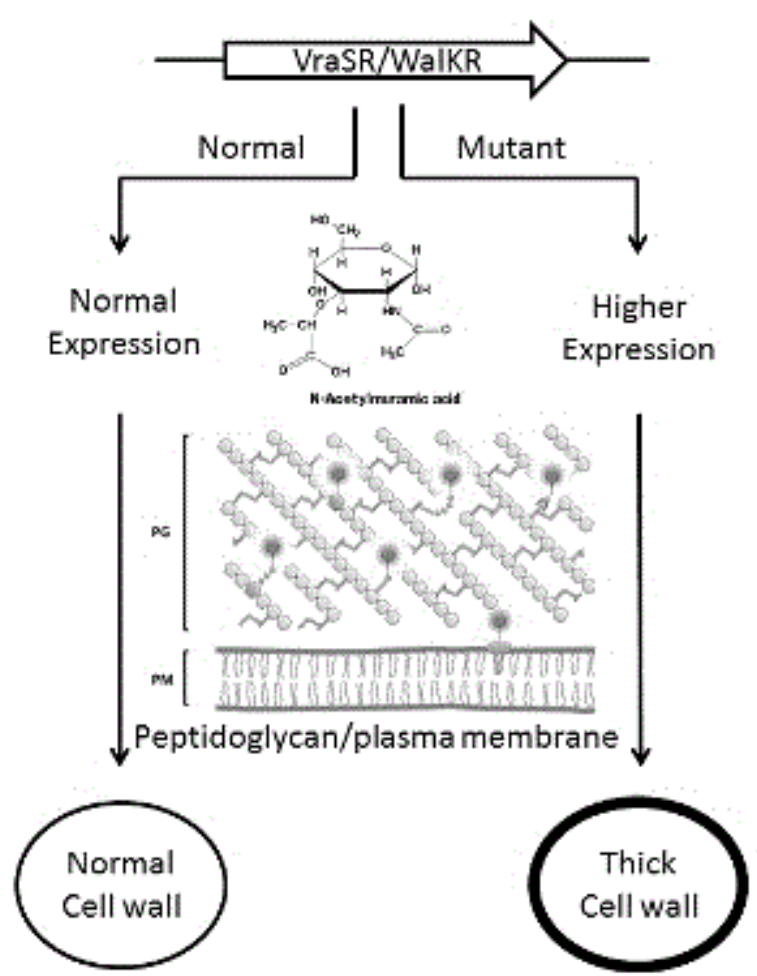

B
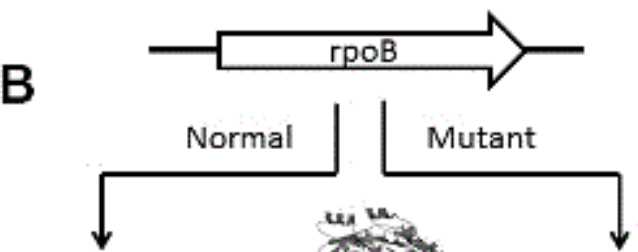

Normal

Expression

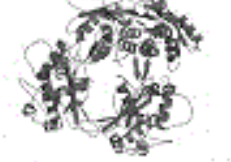

Higher

Expression

RNA polymerase<smiles>C[V]</smiles>

Normal

Expression
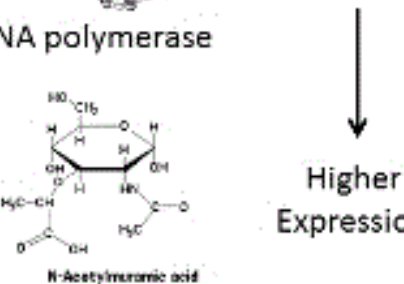

Higher

Expression

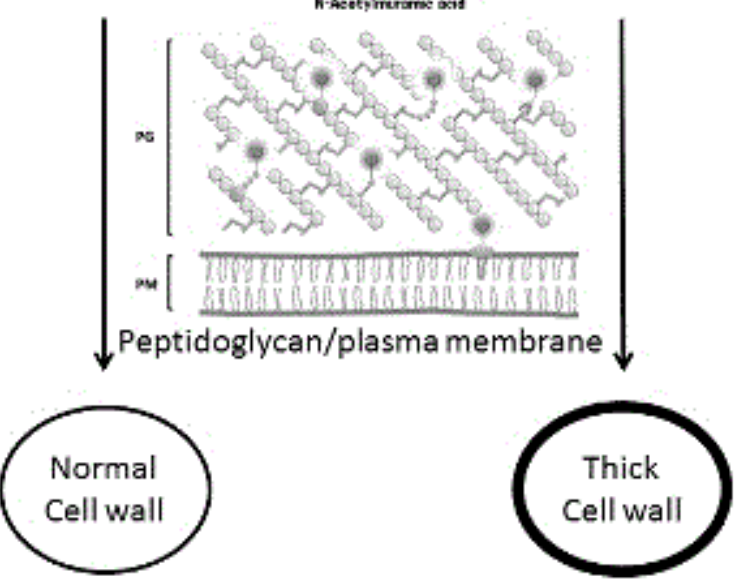

Figure 1: $(A)$ Mutation in vraSR and walKR two component regulator system induce cell wall biogenesis and $(B)$ rpoB gene mutation and cell wall thickening in hVISA/VISA strain. 
Mutation in either of vraS (sensor kinase gene) and vraR (response regulator gene) leads to constitutive activation and over expression of vraSR TCRS. This two component regulatory system (vraSR) is a part operon system vraU-vraT-vraS-vraR and is frequently get mutated in hVISA and VISA clinical strains [7].

In addition, increases the expression of genes encoding enzymes murZ, pbp2, sgtB, tarA, fmtA and lcpC and are involved in cell wall synthesis [8].The vraSR function is determined by vraT/YvqF. A mutation in vraT (Y220C), activate both vraS and vraR and promote upregulation of cell wall biosynthesis. This mutation contributes to the raised level of methicillin and vancomycin resistance. In addition, another variant mutation in vraT (T125I) increases the resistance to both vancomycin and imipenem [9]. Interestingly, up-regulation of two component regulatory system (vraSR) consisting of sensor kinase (vraS) and response regulator (vraR) are induced not only with vancomycin but also found with $\beta$-lactam antibiotics [10]. Kato et al., has performed genetic analysis for the detection of known gene mutation in clinical VISA strains. In this study, 14 (42.4\%) of strains were identified with mutation in either one of the vraT/YvqF, vraS and vraR genes in the four-membered operon system [11].

In walKR TCRS, walK is a sensor histidine kinase and walR response regulator. In addition, TCRS regulate the expression of autolysins. A mutation identified in walK gene, not only increases the vancomycin resistance but also reduce the autolysis of hVISA and VISA strains [12]. Interestingly, among VISA strains rpoB gene mutation was common. The rpoB gene mutation greatly influences the overall transcription of the cell metabolism [13].

Remarkably, mutation in the regulatory gene graR coupled with rpoB mutation translates low-level vancomycin resistant hVISA to high level VISA strains. However, additional studies are essential to establish this association and conversion of hVISA to VISA further [14].

\section{Clinical Significance of Heterogeneous Resistance to Vancomycin in $\boldsymbol{S}$. aureus}

Though the spectrum of infections caused by hVISA/VISA is similar to that of VSSA, the clinical importance of infections remains controversial. It is uncertain whether the hVISA and VISA are more virulent than VSSA isolates and whether their unpredictable level of resistance is responsible for treatment failure [15]. Literature review favours a more favourable outcome for skin and soft tissue infections than nosocomial pneumonia, meningitis, osteomyelitis and endocarditis. It is very difficult to determine the reason for treatment failure if hVISA was detected during the course of infection [16].

In 2003, Moore et al. were able to provide experimental data demonstrated that hVISA was responsible for vancomycin treatment failure in an endocarditis patient. In the study, both pre-treatment and relapse MRSA isolates had MIC of $<2 \mathrm{mg} / \mathrm{ml}$ for vancomycin but the PAP analysis proved that relapse isolate has subpopulations of hVISA. Further testing with rabbit model of endocarditis also proved that the heterogeneous resistance of relapse hVISA isolate was responsible for treatment failure [17].

Even though earlier studies have failed to conclusively identify the relationship between hVISA and poor clinical outcomes, a recent meta-analysis study by Van Hal et al. have reported a 2.37 fold increased risk of vancomycin treatment failure in patients with hVISA than VSSA, although the overall mortality rates for hVISA and VSSA infections was similar [18].

The increasing reports of vancomycin therapy failure with higher MICs and the increasing reports of hVISA isolates prompted the CLSI to revise the breakpoints for vancomycin MIC for $S$. aureus in 2006. The rationale behind this move was that lower susceptible ranges was more likely to exclude the hVISA strains occurring more frequently at higher MICs from the susceptible ranges and hence reducing the possibilities for therapeutic failure. The revisions of the MICs are given in Table 2.

\section{Clinical Markers of hVISA/VISA Infections}

Clinical outcome of hVISA infection varies with the infection site, severity of infection and the presence of any foreign body. Potential markers of hVISA infection were found to be the presence of high bacterial load such as with endocarditis and pneumonia, persistent fever and bacteraemia, worsening of signs of infections after commencement of vancomycin therapy, and presence of prosthetic devices such as orthopedic implants, artificial valves, and pacemakers $[19,20]$.

\begin{tabular}{|c|c|c|c|c|}
\hline \multirow[b]{2}{*}{ Vancomycin interpretation } & \multirow[b]{2}{*}{ Phenotypes } & \multicolumn{3}{|c|}{ Broth Microdilution method (Reference method recommended by CLSI, EUCAST, BSAC, etc) } \\
\hline & & $\begin{array}{l}\text { CLSI interpretation prior to } \\
2006 \text { (in } \mu \mathrm{g} / \mathrm{ml} \text { ) }\end{array}$ & $\begin{array}{l}\text { CLSI interpretation after } 2006 \\
\text { (in } \mu \mathrm{g} / \mathrm{ml} \text { ) }\end{array}$ & $\begin{array}{l}\text { EUCAST interpretation (in } \\
\mu \mathrm{g} / \mathrm{ml} \text { ) }\end{array}$ \\
\hline Susceptible & VSSA & $\leq 4$ & $\leq 2$ & $\leq 2$ \\
\hline *Heteroresistant & *hVISA & - & - & - \\
\hline Intermediate & VISA & 8-16 & $4-8$ & Excluded from the definition \\
\hline Resistant & VRSA & $\geq 32$ & $\geq 16$ & $>2$ \\
\hline
\end{tabular}

Table 2: CLSI and EUCAST breakpoints for vancomycin.

Second antibiotic administered as necessitated by lack of improvement and recurrent BSI within 60 days of vancomycin treatment should also be considered in case of hVISA infections. Prior exposure to vancomycin is one of the considerable factors in clinical outcome analysis [21]. 


\section{Role of Other Factors Contributing to Treatment Failure with hVISA/VISA Strains}

The decreased action of vancomycin on hVISA/VISA may also be attributed to other mutation associated biological features like slower growth rate, reduced autolytic activity, thickened cell wall with reduced peptidoglycan cross-linking [22,23]. Various drug related factors also contribute to the poor outcomes. For example, lethal activity of vancomycin is compromised in the presence of structural communities like biofilms. Vancomycin is also less bactericidal than other antistaphylococcal penicillins and its activity falls between bacteriostatic tetracycline and bactericidal penicillins [24,25]. It is often reflected in the delayed clinical response evidenced by prolonged bacteraemia and persistent fever in MRSA endocarditis patients [26-28].

Thus the vancomycin treatment failure may be due to the enhanced virulence of organism or due to an intrinsic property of the drug or perhaps a combination of both. Further research is needed to find the relevance of hVISA in patients with vancomycin treatment failure. A standard screening test with high sensitivity and specificity for detecting hVISA/VISA needs to be implemented to better understand the prevalence and clinical impact of these organisms [29].

\section{Role of Diagnostic Laboratory in Early Detection of hVISA/VISA}

As heteroresistant subpopulations exists in strains with an MIC of 2 $\mu \mathrm{g} / \mathrm{ml}$, detection of hVISA phenotype and high vancomycin MIC values were directly related [30]. Detection of vancomycin heteroresistance needs careful selection of isolates that requires testing as it is not feasible to test all $S$. aureus isolates. The different methods employed for the detection of heteroresistance are described below.

\section{Population study}

As described by Hiramitsu et al. in 1997, specific inocula of MRSA are plated onto Brain Heart Infusion agar containing vancomycin at a concentration of $4 \mu \mathrm{g} / \mathrm{ml}$. Any visible colony on the plates observed after incubation for 48 hours is considered heteroresistance [31].

\section{Population analysis}

Population analysis profile-area under the curve ratio (PAP-AUC): The colonies to be tested are incubated in Tryptone Soy broth for 24 hours. The colonies (undiluted and in dilutions of $1 / 10^{8}$ and $1 / 10^{5}$ ) are then spiral plated onto several BHI agar plates containing varying concentration of vancomycin from 0.5 to $8 \mu \mathrm{g} / \mathrm{ml}$. A colony count is done on all plates after 48 hours of incubation at $35^{\circ} \mathrm{C}$. The $\mathrm{CFU} / \mathrm{ml}$ and vancomycin concentrations are graded and an area under the curve is obtained. A ratio is obtained by dividing the AUC of test strain with the AUC of the hVISA standard Mu3 strain that is plated along with the test strain. Values of 0.9 to 1.3 is considered hVISA [32].

\section{Screen agars}

A number of vancomycin screen agars have been proposed for the detection of hVISA. However the most popular one is the MHA5T agar which is Mueller Hinton agar incorporated with $5 \mu \mathrm{g} / \mathrm{ml}$ of teicoplanin. A 2 McFarland test suspension is spot inoculated on the plate and reading taken after 48 hours. Any growth is considered positive for hVISA [33].

\section{Modified/Macrodilution E-test}

A 2 McFarland standard suspension of the test strain is made. 200 $\mu \mathrm{l}$ of this suspension is pipetted onto a BHI plate and swabbed evenly. The vancomycin E-test strip is placed on the surface and the plate is incubated at $35^{\circ} \mathrm{C}$ for 48 hours. An MIC of $8 \mu \mathrm{g} / \mathrm{ml}$ or more is taken as heteroresistance [34].

\section{Glycopeptide resistant detection (GRD) E-test}

This method utilises the double sided E-strip containing serially increasing concentrations of vancomycin and teicoplanin on opposing sides and a nutritional supplement. The 0.5 McFarland standard suspension of the test strain is evenly inoculated onto Mueller Hinton agar supplemented with $5 \%$ blood and the E-strip is placed on the inoculum. Reading is taken after 24 and 48 hours of incubation. An MIC of $8 \mu \mathrm{g} / \mathrm{ml}$ or more with vancomycin or teicoplanin is taken as heteroresistance [35].

Detection of hVISA is challenging in clinical microbiology laboratories because reliable and practical method is not available for routine practice. However, MRSA with vancomycin MIC $\leq 2 \mu \mathrm{g} / \mathrm{ml}$ especially $1 \mu \mathrm{g} / \mathrm{ml}-2 \mu \mathrm{g} / \mathrm{ml}$ are more likely to result in vancomycin treatment failure due to hVISA/VISA infection. These isolates with vancomycin MIC of $1 \mu \mathrm{g} / \mathrm{ml}-2 \mu \mathrm{g} / \mathrm{ml}$ are suitable for screening the presence of hVISA phenotypes using macro E-test/GRD E-test. The MRSA isolates identified with either of criteria, Macro E-test (teicoplanin $\geq 12 \mu \mathrm{g} / \mathrm{ml}$ or teicoplanin $\geq 8 \mu \mathrm{g} / \mathrm{ml}$ and vancomycin $\geq 8$ $\mu \mathrm{g} / \mathrm{ml}$ ) and GRD E-test (vancomycin/teicoplanin $\geq 8 \mu \mathrm{g} / \mathrm{ml}$ ) is suggestive of the presence of hVISA phenotype. Further this hVISA isolates should be confirmed by PAP-AUC analysis (Gold standard). PAP-AUC ratio is interpreted as $<0.9$; VSSA, $0.9-1.3$; hVISA, and $>1.3$; VISA (Figure 2).

Optimum detection of hVISA strains relies on testing of higher inoculum $\left[10^{8} \mathrm{CFU}\right.$ for PAP/AUC analysis and $2 \mathrm{McF}$ arland standard inoculum for macromethod $\mathrm{E}$ test], use of more nutritious media like BHIA, MHA supplemented with sheep/horse blood and longer incubation times (48 hrs) [36]. In spite of the availability of several screening assays such as modified E-test methods and screening agars, presence of hVISA strains can be confirmed by PAP/AUC analysis only [37]. Though PAP is labour intensive, costly and time consuming, it still remains the gold standard in assessing the heteroresistant populations.

Clinical markers combined with laboratory screening methods may assist clinicians in reassessing treatment options for hVISA infections. Correlation of in vitro results with efficacy studies in animal models or clinical outcomes is important for screening of the increasing levels of resistance. The duration of bacteremia, complications, and outcome should be correlated with the vancomycin MICs and hVISA status.

\section{Molecular Characterisation of hVISA/VISA}

The specific determinant for molecular characterisation of hVISA/ VISA was not reported. As discussed earlier, mutation in TCRS and rpoB gene results in generation of hVISA/VISA strains. Combined analysis of clinical markers, laboratory diagnosis and screening for the presence of mutation in genes involved in cell metabolism helps to understand the clear mechanism of hVISA/VISA strains. 
Citation: Devi Y, Punithavathy PM, Thomas S, Veeraraghavan B (2015) Challenges in the Laboratory Diagnosis and Clinical Management of Heteroresistant Vancomycin Staphylococcus aureus (hVISA). Clin Microbiol 4: 214. doi:10.4172/2327-5073.1000214

Page 5 of 9

\section{Alternative Therapies for hVISA/VISA Infections}

Vancomycin is the drug of choice for invasive infections caused by multidrug-resistant $S$. aureus most notably nosocomially acquired methicillin-resistant $S$. aureus (MRSA). Clinical cure rate of vancomycin was found to be $35.5 \%$ in patients with MRSA associated nosocomial pneumonia [38]. All reported cases of hVISA and VISA was associated with high bacterial load infections and clinical success rate was found to be $31.8 \%$ for vancomycin and $60 \%$ in case of surgical debridement [39].

Reasons for the decline in efficacy of vancomycin in the treatment of invasive infections was found to be due to poor tissue and intracellular penetration of the drug, slow bactericidal effect, lack of interference with toxin production, loss of accessory gene-regulator function in MRSA [40,41]. Increasing reports of in vitro resistance and treatment failures due to hVISA/VISA strains emphasize the need for newer therapeutic options. Administration of alternative therapies in case of treatment failure should be based on both clinical and laboratory findings. In addition to the treatment failure, other contributing factors includes adequate dose administration with less toxicity, trough levels monitoring remain as challenges in usage of vancomycin.

Fortunately, several other alternatives for treating hVISA/VISA infections include linezolid, quinupristin-dalfopristin, daptomycin, and tigecycline. All these are approved approved by the FDA. Though in vitro and animal studies data were available, clinical trials based efficacy rate was not released for the above mentioned newer antimicrobial agents.

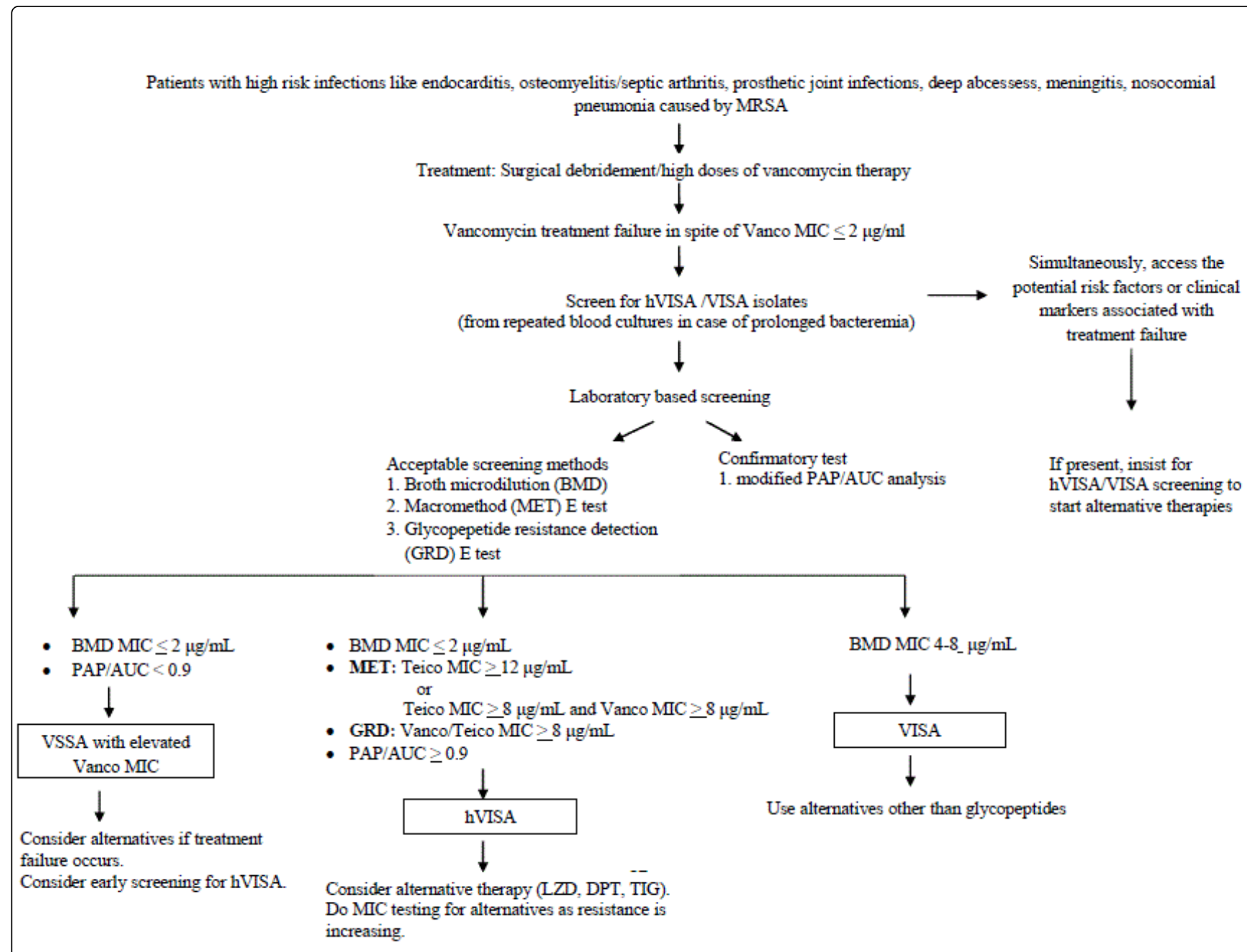

Figure 2: Clinical and laboratory based algorithms for detection of hVISA/VISA.

\section{Rifampin and fusidic acid}

Combination therapy with rifampin and fusidic acid was found to possess good in vitro and in-vivo activity against multidrug resistant $S$. aureus. These drugs should always be used in combination with other anti-staphylococcal agent, as resistance develops rapidly with monotherapy $[42,43]$. Oral rifampin and fusidic acid was reported to be successful against hVISA/VISA infections in patients with vancomycin treatment failure. 


\section{Linezolid}

Linezolid, a completely synthetic oxazolidinone can be used for the therapy of $S$. aureus with reduced vancomycin susceptibility. Linezolid acts by inhibiting protein synthesis by binding with $50 \mathrm{~S}$ ribosome. It is a bacteriostatic agent with good action against MRSA, hVISA and VISA infections, including high bacterial load infections like endocarditis, pneumonia, skin and soft tissue infections [44]. Linezolid is especially preferred for treating MRSA/hVISA/VISA pneumonia/ lower respiratory tract infection (LRTI). It is also useful in treating patients not responding to vancomycin. Development of linezolid resistance against $S$. aureus is of great concern. A novel linezolid resistance (LRSA) mechanism carried on a mobile genetic element has been reported recently in cfr-constitutive LRSA outbreak in Madrid $[45,46]$. As high rate of toxicity has been found in linezolid administered patients, it is advisable to use it with caution in prolonged therapies.

\section{Daptomycin}

Daptomycin, a cyclic lipopeptide should be used with caution against high bacterial load $S$. aureus infection [47]. Daptomycin acts directly against cell membrane through $\mathrm{ca}^{2+}$ dependent mechanism results in efflux of potassium and inhibition of protein, DNA and RNA synthesis. For high bacterial load infection the recommended dosage for daptomycin is $6 \mathrm{mg} / \mathrm{kg} /$ day and for SSTIs are $4 \mathrm{mg} / \mathrm{kg} /$ day. In vitro animal studies have suggested that daptomycin possess slower rate of bactericidal activity against hVISA than VSSA [48]. Cross-resistance between daptomycin resistance and hVISA/VISA are reported in a patient with endocarditis [49]. The highest rate of daptomycin nonsusceptibility was reported in $15 \%$ of hVISA and $38 \%$ of VISA strains. High dose daptomycin $(10 \mathrm{mg} / \mathrm{kg} /$ day $)$ with trimethoprimsulfamethoxazole has shown to be promising for traetemnet of hVISA/ VISA infections [50].

\section{Quinupristin-dalfopristin and tigecycline}

Quinupristin-dalfopristin (QD), a combination of two streptogramins has potent in vitro activity against a range of hVISA, VISA and MRSA strains. It acts by inducing conformational changes in the ribosome. The recommended dosage is $7.5 \mathrm{mg} / \mathrm{kg} \mathrm{q} 8 \mathrm{~h}-12 \mathrm{~h}$ for skin and soft tissue infection, bone and joint infections. The MIC 90 for hVISA and VISA strains was found to be $1 \mu \mathrm{g} / \mathrm{ml}$ [51].

Tigecycline belongs to tetracycline family and was the first glycylcycline used in clinical cases. Tigecycline binds with 30 S subunit of ribosome and inhibit protein synthesis. It is active against MSSA and MRSA strains and small numbers of VISA strains with MIC90 of $0.12 \mu \mathrm{g} / \mathrm{ml}$ to $1 \mu \mathrm{g} / \mathrm{ml}[52,53]$.

\section{Newer antimicrobials against hVISA/VISA infections in development}

Dalbavancin, a semisynthetic lipoglycopeptide has good in vitro activity against $S$. aureus with MIC $0.015 \mu \mathrm{g} / \mathrm{ml}$ to $0.5 \mu \mathrm{g} / \mathrm{ml}$ by inhibiting cell wall synthesis. It is effective in patients infected with $S$. aureus that are resistant to vancomycin, linezolid and quinupristindalfopristin [54]. MICs was found to be higher for hVISA/VISA strains $(0.06 \mu \mathrm{g} / \mathrm{ml}$ to $2 \mu \mathrm{g} / \mathrm{ml})$ and thus higher concentrations was required to achieve in vivo killing. Its efficacy against VISA strains have also been demonstrated in animal models [55]. Dalbavancin is given as $1000 \mathrm{mg}$ intravenously on dayl and followed by $500 \mathrm{mg}$ on day 8 sustains the plasma concentration to $20 \mu \mathrm{g} / \mathrm{ml}$ [56].

Oritavancin, a lipoglycopeptide possess notable in vitro activity against VRSA strains and reduced bactericidal activity against VISA strains by disrupting the cell membrane [57,58]. Telavancin, a semisynthetic lipoglycopeptide and derivative of vancomycin has MIC ranging between 0.03 and $1 \mu \mathrm{g} / \mathrm{ml}$ against methicillin resistant $S$. aureus strains, and 0.125 to $1 \mu \mathrm{g} / \mathrm{ml}$ for VISA strains [59-61].

Newer cephalosporins like ceftaroline and ceftobiprole possess good in vitro activity against hVISA and VISA strains with MIC range of $0.25 \mu \mathrm{g} / \mathrm{ml}$ to $4 \mu \mathrm{g} / \mathrm{ml}$ and $<2 \mu \mathrm{g} / \mathrm{ml}$ respectively [62]. Through animal studies, ceftaroline was found to be superior to vancomycin, linezolid, teicoplanin, and arbekacin in treatment of MRSA infections [63]. In 2005, Jacqueline et al. reported that ceftaroline has high bactericidal activity against hVISA compared with linezolid/vancomycin treatment [64]. Similarly, ceftobiprole is also reported to have high bactericidal activity against hVISA strains than vancomycin [65].

Though various studies have reported the successful therapies against hVISA/VISA infections with alternative antimicrobials, potential concerns including toxicity with prolonged linezolid use, cross-resistance with lipoglycopeptides, and low-level daptomycin non-susceptibility should be taken into consideration. To overcome these shortcomings, vancomycin is combined with other alternatives in treatment of severe MRSA infections. Reasons behind the combination therapy includes broadening the spectrum of antistaphylococcal activity to include VISA/hVISA strains, preventing the emergence of $S$. aureus with reduced vancomycin susceptibility, enhancing bactericidal activity, inhibiting toxin production and facilitating the penetration into cells and tissues not reached by vancomycin.

\section{Successfully Treated hVISA/VISA Cases: Combination Therapy}

The combination of vancomycin or daptomycin and a $\beta$-lactam antibiotic were also studied for treating hVISA/VISA infection. The synergistic activity of $\beta$-lactam including ceftaroline, cefazolin, and piperacillin-tazobactam has been evaluated with vancomycin or daptomycin for therapy [66-68]. Addition of $\beta$-lactam antibiotic to either vancomycin or daptomycin enhances the activity of vancomycin/daptomycin by interacting with cell wall synthesis. However, further studies are warranted to elucidate the interaction in improved killing of MRSA.

In patient with MRSA bacteremia and are failing with vancomycin therapy were successfully treated with linezolid in combination with rifampin plus fusidic acid or linezolid alone or with trimethoprimsulfamethoxazole (orally $160 \mathrm{mg}$ and $800 \mathrm{mg}$ daily). Studies have reported those hVISA/VISA endocarditis were recovered from infection with an alternative therapy of linezolid alone or in combination with rifampin or fusidic acid $[69,70]$.

For patient with acute MRSA cholecystitis, initially treated with vancomycin for 10 weeks results in poor outcome and hVISA development with an MIC of $8 \mu \mathrm{g} / \mathrm{ml}$ was documented. This patient was successfully treated with the combination of linezolid $(600 \mathrm{mg}$ twice a day) plus trimethoprim-sulfamethoxazole $(160 \mathrm{mg}$ and 800 $\mathrm{mg}$ ) plus doxycycline (100 mg orally) twice a day [71]. Similarly in patient with septic arthritis, developed hVISA and treatment failure was completely recovered while treated with linezolid alone or 
linezolid plus rifampin and fusidic acid [70]. Patient with endocarditis who failed with both vancomycin and daptomycin therapy were successfully treated with linezolid (600 $\mathrm{mg} \mathrm{q} 12 \mathrm{hrs)}$ and fusidic acid (500mg q 8hrs) or linezolid with trimethoprim-sulfamethoxazole plus quinupristin-dalfopristin [71].

\section{Conclusion}

Presence of hVISA/VISA strains in serious MRSA infections may lead to treatment failure. As the therapeutic options were limited, appropriate use of vancomycin prevents the development of vancomycin resistance. Optimal detection methods are essential for proper guidance of therapeutic decision making and complete understanding the impact of hVISA infection. The clinician can continue to use vancomycin as recommended by Infectious Diseases Society of America guidelines. Except, for patients who are not responding to vancomycin and present with risk factors of hVISA/ VISA infections, alternative therapies can be considered. Treating with daptomycin should be used with caution for hVISA/VISA infection, because of the potential for cross resistance.

Early detection/surveillance of hVISA/VISA strains and standard infection control practices for the prevention of spread of resistance strains should be strictly followed in all hospital settings. Detection of mutation involving with TCRS and rpoB gene for development of heteroresistance may facilitate earlier identification of hVISA/VISA strains and thus its associated treatment failure. Even though alternatives were available against hVISA/VISA infections, treatment guidelines have to be redefined with caution as only limited information are available.

\section{References}

1. Pootoolal J, Neu J, Wright GD (2002) Glycopeptide antibiotic resistance. Annu Rev Pharmacol Toxicol 42: 381-408.

2. Tenover FC, Moellering RC Jr (2007) The rationale for revising the Clinical and Laboratory Standards Institute vancomycin minimal inhibitory concentration interpretive criteria for Staphylococcus aureus. Clin Infect Dis 44: 1208-1215.

3. Plipat N, Livni G, Bertram H, Thomson RB Jr (2005) Unstable vancomycin heteroresistance is common among clinical isolates of methiciliin-resistant Staphylococcus aureus. J Clin Microbiol 43: 2494-2496.

4. Cui L, Ma X, Sato K, Okuma K, Tenover FC, et al. (2003) Cell wall thickening is a common feature of vancomycin resistance in Staphylococcus aureus. J Clin Microbiol 41: 5-14.

5. Rybak MJ, Cappelletty DM, Moldovan T, Aeschlimann JR, Kaatz GW (1998) Comparative in vitro activities and postantibiotic effects of the oxazolidinone compounds eperezolid (PNU-100592) and linezolid (PNU-100766) versus vancomycin against Staphylococcus aureus, coagulase-negative staphylococci, Enterococcus faecalis, and Enterococcus faecium. Antimicrob Agents Chemother 42: 721-724.

6. Reynolds PE1 (1989) Structure, biochemistry and mechanism of action of glycopeptide antibiotics. Eur J Clin Microbiol Infect Dis 8: 943-950.

7. Boyle-Vavra S, Yin S, Jo DS, Montgomery CP, Daum RS (2013) VraT/ $\mathrm{YvqF}$ is required for methicillin resistance and activation of the VraSR regulon in Staphylococcus aureus. Antimicrob Agents Chemother 57: 83-95.

8. Yin S, Daum RS, Boyle-Vavra S (2006) VraSR two-component regulatory system and its role in induction of pbp 2 and vraSR expression by cell wall antimicrobials in Staphylococcus aureus. Antimicrob Agents Chemother 50: 336-343.

9. Kato Y, Suzuki T, Ida T, Maebashi K (2010) Genetic changes associated with glycopeptide resistance in Staphylococcus aureus: predominance of amino acid substitutions in YvqF/VraSR. J Antimicrob Chemother 65: 37-45.

10. Shoji M, Cui L, Iizuka R, Komoto A, Neoh HM, et al. (2011) walK and clpP mutations confer reduced vancomycin susceptibility in Staphylococcus aureus. Antimicrob Agents Chemother 55: 3870-3881.

11. Saito M, Katayama Y, Hishinuma T, Iwamoto A, Aiba Y, et al. (2014) "Slow VISA," a novel phenotype of vancomycin resistance, found in vitro in heterogeneous vancomycin-intermediate Staphylococcus aureus strain Mu3. Antimicrob Agents Chemother 58: 5024-5035.

12. Matsuo M, Hishinuma T, Katayama Y, Cui L, Kapi M, et al. (2011) Mutation of RNA polymerase beta subunit (rpoB) promotes hVISA-toVISA phenotypic conversion of strain Mu3. Antimicrob Agents Chemother 55: 4188-4195.

13. Liu C, Chambers HF (2003) Staphylococcus aureus with heterogeneous resistance to vancomycin: epidemiology, clinical significance, and critical assessment of diagnostic methods. Antimicrob Agents Chemother 47: 3040-3045.

14. Holmes NE, Johnson PD, Howden BP (2012) Relationship between vancomycin-resistant Staphylococcus aureus, vancomycin-intermediate S. aureus, high vancomycin MIC, and outcome in serious S. aureus infections. J Clin Microbiol 50: 2548-2552.

15. Moore MR, Perdreau-Remington F, Chambers HF (2003) Vancomycin treatment failure associated with heterogeneous vancomycinintermediate Staphylococcus aureus in a patient with endocarditis and in the rabbit model of endocarditis. Antimicrob Agents Chemother 47: 1262-1266.

16. van Hal SJ, Jones M, Gosbell IB, Paterson DL (2011) Vancomycin heteroresistance is associated with reduced mortality in ST239 methicillin-resistant Staphylococcus aureus blood stream infections. PLoS One 6: e21217.

17. Charles PG, Ward PB, Johnson PD, Howden BP, Grayson ML (2004) Clinical features associated with bacteremia due to heterogeneous vancomycin-intermediate Staphylococcus aureus. Clin Infect Dis 38: $448-451$

18. Dhand A, Sakoulas G (2012) Reduced vancomycin susceptibility among clinical Staphylococcus aureus isolates ('the MIC Creep'): implications for therapy. F1000 Med Rep 4: 4.

19. Tarai B, Das P, Kumar D1 (2013) Recurrent Challenges for Clinicians: Emergence of Methicillin-Resistant Staphylococcus aureus, Vancomycin Resistance, and Current Treatment Options. J Lab Physicians 5: 71-78.

20. Amatya R, Devkota P, Gautam A (2014) Reduced susceptibility to vancomycin in methicillin resistant staphylococcus aureus: a time for action. Nepal Med Coll J 16: 42-44.

21. Appelbaum PC1 (2007) Reduced glycopeptide susceptibility in methicillin-resistant Staphylococcus aureus (MRSA). Int J Antimicrob Agents 30: 398-408.

22. Jones RN1 (2006) Microbiological features of vancomycin in the 21st century: minimum inhibitory concentration creep, bactericidal/static activity, and applied breakpoints to predict clinical outcomes or detect resistant strains. Clin Infect Dis 42 Suppl 1: S13-24.

23. Rybak MJ1 (2006) The pharmacokinetic and pharmacodynamic properties of vancomycin. Clin Infect Dis 42 Suppl 1: S35-39.

24. Maor Y, Hagin M, Belausov N, Keller N, Ben-David D, et al. (2009) Clinical features of heteroresistant vancomycin-intermediate Staphylococcus aureus bacteremia versus those of methicillin-resistant $S$. aureus bacteremia. J Infect Dis 199: 619-624.

25. Cosgrove SE, Fowler VG Jr (2008) Management of methicillin-resistant Staphylococcus aureus bacteremia. Clin Infect Dis 46 Suppl 5: S386-393.

26. Sakoulas G, Gold HS, Cohen RA, Venkataraman L, Moellering RC, et al. (2006) Effects of prolonged vancomycin administration on methicillinresistant Staphylococcus aureus (MRSA) in a patient with recurrent bacteraemia. J Antimicrob Chemother 57: 699-704.

27. Pitz AM, Yu F, Hermsen ED, Rupp ME, Fey PD, et al. (2011) Vancomycin susceptibility trends and prevalence of heterogeneous vancomycin-intermediate Staphylococcus aureus in clinical methicillinresistant S. aureus isolates. J Clin Microbiol 49: 269-274. 
28. Wang JL, Lai CH, Lin HH, Chen WF, Shih YC, et al. (2013) High vancomycin minimum inhibitory concentrations with heteroresistant vancomycin-intermediate Staphylococcus aureus in meticillin-resistant $S$. aureus bacteraemia patients. Int J Antimicrob Agents 42: 390-394.

29. Hiramatsu K, Aritaka N, Hanaki H, Kawasaki S, Hosoda Y, et al. (1997) Dissemination in Japanese hospitals of strains of Staphylococcus aureus heterogeneously resistant to vancomycin. Lancet 350: 1670-1673.

30. Wootton M, Howe RA, Hillman R, Walsh TR, Bennett PM, et al. (2001) A modified population analysis profile (PAP) method to detect heteroresistance to vancomycin in Staphylococcus aureus in a UK hospital. J Antimicrob Chemother 47: 399-403.

31. Wootton M, MacGowan AP, Walsh TR, Howe RA (2007) A multicenter study evaluating the current strategies for isolating Staphylococcus aureus strains with reduced susceptibility to glycopeptides. J Clin Microbiol 45: 329-332.

32. Walsh TR, Bolmström A, Qwärnström A, Ho P, Wootton M, et al. (2001) Evaluation of current methods for detection of staphylococci with reduced susceptibility to glycopeptides. J Clin Microbiol 39: 2439-2444.

33. Leonard SN, Rossi KL, Newton KL, Rybak MJ (2009) Evaluation of the Etest GRD for the detection of Staphylococcus aureus with reduced susceptibility to glycopeptides. J Antimicrob Chemother 63: 489-492.

34. van Hal SJ, Wehrhahn MC, Barbagiannakos T, Mercer J, Chen D, et al. (2011) Performance of various testing methodologies for detection of heteroresistant vancomycin-intermediate Staphylococcus aureus in bloodstream isolates. J Clin Microbiol 49: 1489-1494.

35. Riederer K, Shemes S, Chase P, Musta A, Mar A, et al. (2011) Detection of intermediately vancomycin-susceptible and heterogeneous Staphylococcus aureus isolates: comparison of Etest and Agar screening methods. J Clin Microbiol 49: 2147-2150.

36. Wunderink RG, Rello J, Cammarata SK, Croos-Dabrera RV, Kollef MH (2003) Linezolid vs vancomycin: analysis of two double-blind studies of patients with methicillin-resistant Staphylococcus aureus nosocomial pneumonia. Chest 124: 1789-1797.

37. Drew RH (2007) Emerging options for treatment of invasive, multidrugresistant Staphylococcus aureus infections. Pharmacotherapy 27: 227-249.

38. Micek ST (2007) Alternatives to vancomycin for the treatment of methicillin-resistant Staphylococcus aureus infections. Clin Infect Dis 45 Suppl 3: S184-190.

39. van Hal SJ, Fowler VG Jr (2013) Is it time to replace vancomycin in the treatment of methicillin-resistant Staphylococcus aureus infections? Clin Infect Dis 56: 1779-1788.

40. Zinn CS, Westh H, Rosdahl VT; Sarisa Study Group (2004) An international multicenter study of antimicrobial resistance and typing of hospital Staphylococcus aureus isolates from 21 laboratories in 19 countries or states. Microb Drug Resist 10: 160-168.

41. Howden BP (2005) Recognition and management of infections caused by vancomycin-intermediate Staphylococcus aureus (VISA) and heterogenous VISA (hVISA). Intern Med J 35 Suppl 2: S136-140.

42. Andrade-Baiocchi S, Tognim MC, Baiocchi OC, Sader HS (2003) Endocarditis due to glycopeptide-intermediate Staphylococcus aureus: case report and strain characterization. Diagn Microbiol Infect Dis 45: 149-152.

43. Sánchez García M, De la Torre MA, Morales G, Peláez B, Tolón MJ, et al. (2010) Clinical outbreak of linezolid-resistant Staphylococcus aureus in an intensive care unit. JAMA 303: 2260-2264.

44. Gu B, Kelesidis T, Tsiodras S, Hindler J, Humphries RM (2013) The emerging problem of linezolid-resistant Staphylococcus. J Antimicrob Chemother 68: 4-11.

45. Sharma M, Riederer K, Chase P, Khatib R (2008) High rate of decreasing daptomycin susceptibility during the treatment of persistent Staphylococcus aureus bacteremia. Eur J Clin Microbiol 27: 433-437.

46. Leonard SN, Rybak MJ (2009) Evaluation of vancomycin and daptomycin against methicillin-resistant Staphylococcus aureus and heterogeneously vancomycin-intermediate $S$. aureus in an in vitro pharmacokinetic/pharmacodynamic model with simulated endocardial vegetations. J Antimicrob Chemother 63: 155-160.

47. Boucher HW, Sakoulas G (2007) Perspectives on Daptomycin resistance, with emphasis on resistance in Staphylococcus aureus. Clin Infect Dis 45: 601-608.

48. Yu R, Dale SE, Yamamura D, Stankus V, Lee C (2012) Daptomycinnonsusceptible, vancomycin-intermediate, methicillin-resistant Staphylococcus aureus endocarditis. Can J Infect Dis 23: e48-e50.

49. Jevitt LA, Smith AJ, Williams PP, Raney PM, McGowan JE Jr, et al. (2003) In vitro activities of Daptomycin, Linezolid, and QuinupristinDalfopristin against a challenge panel of Staphylococci and Enterococci, including vancomycin-intermediate staphylococcus aureus and vancomycin-resistant Enterococcus faecium. Microb Drug Resist 9: 389-393.

50. Howden BP, Davies JK, Johnson PDR, Stinear TP, Grayson ML (2010) Reduced vancomycin susceptibility in Staphylococcus aureus, including vancomycin-intermediate and heterogeneous vancomycin-intermediate strains: resistance mechanisms, laboratory detection, and clinical implications. Clin Microbiol Rev 23: 99-139.

51. Petersen PJ, Bradford PA, Weiss WJ, Murphy TM, Sum PE, et al. (2002) In vitro and in vivo activities of tigecycline (GAR-936), daptomycin, and comparative antimicrobial agents against glycopeptide-intermediate Staphylococcus aureus and other resistant gram-positive pathogens. Antimicrob Agents Chemother 46: 2595-2601.

52. Streit JM, Sader HS, Fritsche TR, Jones RN (2005) Dalbavancin activity against selected populations of antimicrobial-resistant Gram-positive pathogens. Diagn Microbiol Infect Dis 53: 307-310.

53. Lefort A, Pavie J, Garry L, Chau F, Fantin B (2004) Activities of dalbavancin in vitro and in a rabbit model of experimental endocarditis due to Staphylococcus aureus with or without reduced susceptibility to vancomycin and teicoplanin. Antimicrob Agents Chemother 48: 1061-1064.

54. Chen AY, Zervos MJ, Vazquez JA (2007) Dalbavancin: a novel antimicrobial. Int J Clin Pract 61: 853-863.

55. Mendes RE, Farrell DJ, Sader HS, Jones RN (2012) Oritavancin microbiologic features and activity results from the surveillance program in the United States. Clin Infect Dis 54 Suppl 3: S203-213.

56. McKay GA, Beaulieu S, Arhin FF, Belley A, Sarmiento I, et al. (2009) Time-kill kinetics of oritavancin and comparator agents against Staphylococcus aureus, Enterococcus faecalis and Enterococcus faecium. J Antimicrob Chemother 63: 1191-1199.

57. Leuthner KD, Cheung CM, Rybak MJ (2006) Comparative activity of the new lipoglycopeptide telavancin in the presence and absence of serum against 50 glycopeptide non-susceptible staphylococci and three vancomycin-resistant Staphylococcus aureus. J Antimicrob Chemother 58: 338-343.

58. Smith WJ, Drew RH (2009) Telavancin: a new lipoglycopeptide for grampositive infections. Drugs Today (Barc) 45: 159-173.

59. Charneski L, Patel PN, Sym D (2009) Telavancin: a novel lipoglycopeptide antibiotic. Ann Pharmacother 43: 928-938.

60. Duplessis C, Crum-Cianflone NF (2011) Ceftaroline: A New Cephalosporin with Activity against Methicillin-Resistant Staphylococcus aureus (MRSA). Clin Med Rev Ther 3.

61. Bogdanovich T, Ednie LM, Shapiro S, Appelbaum PC (2005) Antistaphylococcal activity of ceftobiprole, a new broad-spectrum cephalosporin. Antimicrob Agents Chemother 49: 4210-4219.

62. Jacqueline C, Caillon J, Le Mabecque V, Miègeville A-F, Hamel A, et al. (2007) In vivo efficacy of ceftaroline (PPI-0903), a new broad-spectrum cephalosporin, compared with linezolid and vancomycin against methicillin-resistant and vancomycin-intermediate Staphylococcus aureus in a rabbit endocarditis model. Antimicrob Agents Chemother 51: 3397-3400.

63. Chambers HF (2005) Evaluation of ceftobiprole in a rabbit model of aortic valve endocarditis due to methicillin-resistant and vancomycinintermediate Staphylococcus aureus. Antimicrob Agents Chemother 49: 884-888. 
Citation: Devi Y, Punithavathy PM, Thomas S, Veeraraghavan B (2015) Challenges in the Laboratory Diagnosis and Clinical Management of Heteroresistant Vancomycin Staphylococcus aureus (hVISA). Clin Microbiol 4: 214. doi:10.4172/2327-5073.1000214

Page 9 of 9

64. Werth BJ, Vidaillac C, Murray KP, Newton KL, Sakoulas G, et al. (2013) Novel combinations of vancomycin plus ceftaroline or oxacillin against methicillin-resistant vancomycin-intermediate Staphylococcus aureus (VISA) and heterogeneous VISA. Antimicrob Agents Chemother 57: 2376-2379.

65. Hagihara M, Wiskirchen DE, Kuti JL, Nicolau DP (2012) In vitro pharmacodynamics of vancomycin and cefazolin alone and in combination against methicillin-resistant Staphylococcus aureus. Antimicrob Agents Chemother 56: 202-207.

66. Dilworth TJ, Leonard SN, Vilay AM, Mercier RC (2014) Vancomycin and piperacillin-tazobactam against methicillin-resistant Staphylococcus aureus and vancomycin-intermediate Staphylococcus aureus in an in vitro pharmacokinetic/pharmacodynamic model. Clin Ther 36: 1334-1344.

67. Smith TL, Pearson ML, Wilcox KR, Cruz C, Lancaster MV, et al. (1999) Emergence of vancomycin resistance in Staphylococcus aureus. Glycopeptide-Intermediate Staphylococcus aureus Working Group. N Engl J Med 340: 493-501.
68. Howden BP, Ward PB, Charles PGP, Korman TM, Fuller A, et al. (2004) Treatment outcomes for serious infections caused by methicillinresistant Staphylococcus aureus with reduced vancomycin susceptibility. Clin Infect Dis 38: 521-528.

69. Hageman JC, Pegues DA, Jepson C, Bell RL, Guinan M, et al. (2001) Vancomycin-intermediate Staphylococcus aureus in a home health-care patient. Emerg Infect Dis 7: 1023-1025.

70. Huang YT, Hsiao CH, Liao CH, Lee CW, Hsueh PR (2008) Bacteremia and infective endocarditis caused by a non-daptomycin-susceptible, vancomycin-intermediate, and methicillin-resistant Staphylococcus aureus strain in Taiwan. J Clin Microbiol 46: 1132-1136.

71. Tenover FC, Sinner SW, Segal RE, Huang V, Alexandre SS, et al. (2009) Characterisation of a Staphylococcus aureus strain with progressive loss of susceptibility to vancomycin and daptomycin during therapy. Int J Antimicrob Agents 33: 564-568. 